\title{
Spin-constrained orbital-angular-momentum control in high-harmonic generation
}

\author{
F. Kong, ${ }^{1,2}$ C. Zhang $\odot,{ }^{1,2, *}$ H. Larocque, ${ }^{1}$ F. Bouchard,${ }^{1}$ Z. Li, ${ }^{3}$ M. Taucer, ${ }^{1,2}$ G. Brown, ${ }^{1,2}$ Stefano Severino, ${ }^{4}$ \\ T. J. Hammond, ${ }^{1,2,5}$ E. Karimi, ${ }^{1,6}$ and P. B. Corkum ${ }^{1,2}$ \\ ${ }^{1}$ Department of Physics, University of Ottawa, 25 Templeton Street, Ottawa, Ontario, Canada K1N 6N5 \\ ${ }^{2}$ Joint Attosecond Science Laboratory, University of Ottawa and National Research Council of Canada, \\ 100 Sussex Drive, Ottawa, Ontario, Canada K1A OR6 \\ ${ }^{3}$ School of Optical and Electronic Information, Huazhong University of Science and Technology, Wuhan 430074, China \\ ${ }^{4}$ Dipartimento di Fisica "Ettore Pancini," Università di Napoli Federico II, Complesso Universitario di Monte S. Angelo, \\ Via Cintia, I-80126 Napoli, Italy \\ ${ }^{5}$ Department of Physics, University of Windsor, Windsor, Ontario, Canada N9B 3P4 \\ ${ }^{6}$ Department of Physics, Institute for Advanced Studies in Basic Sciences, Zanjan 45137-66731, Iran
}

(Received 18 October 2018; revised manuscript received 24 April 2019; published 28 October 2019)

\begin{abstract}
The interplay between spin and orbital angular momentum in the up-conversion process allows us to control the macroscopic wave front of high harmonics by manipulating the microscopic polarizations of the driving field. We demonstrate the control of orbital angular momentum in high-harmonic generation from both solidand gas-phase targets using the selection rules of spin angular momentum. The gas-phase harmonics extend the control of angular momentum to the extreme-ultraviolet wavelength. We also propose a bicolor scheme to produce spectrally separated extreme-ultraviolet radiation carrying orbital angular momentum.
\end{abstract}

DOI: 10.1103/PhysRevResearch.1.032008

Structuring the spatial profiles of electromagnetic fields can lead to important physics and applications [1,2]. Two physical quantities in particular are the phase front and the polarization and have garnered great attention recently [3]. Shaping the wave front of extreme-ultraviolet (XUV) or soft $\mathrm{x}$-ray radiation is challenging due to the limitation of available optics $[4,5]$ despite the demand for these wavelengths in microscopy, spectroscopy, and lithography applications [6-8]. The wave-front shaping can also be achieved by modifying the generating scheme, for example, a spiral optical beam can be generated from free-electron lasers by modifying the undulator $[9,10]$. With a table-top apparatus, a nonlinear frequency conversion under nonperturbative conditions provides a feasible route to control or transfer the structured wave fronts to the XUV and even x-ray regions. By preshaping the driving optical field, the phase spirals that define the orbital angular momentum (OAM) can be imparted to the generated frequencies $[6-8,11,12]$. Similarly, the local polarization or the spin angular momentum (SAM) of the XUV field can also be controlled by engineering the polarization of the incident driving fundamental beam [13-15]. The circularly polarized XUV beams are a source for polarization-sensitive measurements of inner-shell electrons in materials and distinguishing chiral molecules [16-18].

\footnotetext{
*Corresponding author: chunmei.zhang@uottawa.ca

Published by the American Physical Society under the terms of the Creative Commons Attribution 4.0 International license. Further distribution of this work must maintain attribution to the author(s) and the published article's title, journal citation, and DOI.
}

Previous research shows that, on their own, the spin and the orbital angular momentum are conserved during the nonlinear conversions in both perturbative [19-21] and nonperturbative regimes $[11,12,22]$ under paraxial conditions. These studies either modify the polarization or the wave front of the driving laser beams, while keeping the other part uniform or planar. Such "pure mode" configurations concentrate on studying the behavior of either the spin or the orbital angular momentum during the nonlinear conversion and deliberately eliminate possible influences from the other part of the angular momentum.

The interaction involving both spin and orbital angular momentum are discussed within the framework of perturbative nonlinear optics and metasurfaces, where both the polarization and the phase front of the fundamental beam are shaped $[23,24]$. So far, however, there has been little discussion about the interplay between these two types of angular momentum in high-order nonlinearities, which would allow us to further control the angular momentum in extremely short wavelengths. Indeed, the mechanism of generating highorder harmonics at XUV wavelengths is very different from ordinary nonlinear optics. Our study links strong-field physics to beam-shaping techniques and opens up another research direction in nonperturbative nonlinear interactions.

Instead of using a driving beam with a single spatial mode or a uniform polarization state, we manipulate both the spin and orbital angular momentum of the driving beam to control the orbital angular momentum of the generated harmonic by means of spin selection rules. In our specific case, the spin $(s= \pm 1)$ selection rule eliminates the channels that could lead to higher OAM states [11,7], confining the OAM of the high harmonics to that of the fundamental, in our case \pm 1 , unit. The collinear geometry of our approach ensures efficient 
high-harmonic production as compared to previous methods $[6,8]$. This interplay between the spin and orbital angular momentum during up-conversion allows us to control the macroscopic wave front in the short-wavelength radiation by manipulating the microscopic polarization of the driving field.

The experiments are performed with both solid and gas targets. The solid target gives us access to both above and below band-gap harmonics, which can be easily manipulated and measured with conventional optics due to their low frequency. Harmonics generated from noble gases, on the other hand, allow this technique to be transferred to much higher frequencies. We will show that our results for photon energy $\sim 42 \mathrm{eV}$ are consistent with a beam with OAM values equal to \pm 1 .

In our experiment a laser beam (with a duration of $50 \mathrm{fs}$, and a center wavelength of $1.8 \mu \mathrm{m}$ for the $\mathrm{ZnO}$ crystal and $800 \mathrm{~nm}$ for argon gas) generates harmonics in a solid- or gas-phase target. Figure 1(a) shows the experimental setup of generating structured high-harmonic beams from a solid crystal target and interferometric characterization of the OAM on the harmonic beams. We shape the spatial characteristics of the beam with a $q$-plate, a liquid-crystal device that can change the polarization (or phase) of the incident laser beam [25-27] point by point across the beam profile. After passing through the $q$-plate $(q=1 / 2)$, our 1.8 - $\mu \mathrm{m}$ beam in the Laguerre-Gaussian $\left(\mathrm{LG}_{0,0}\right)$ mode is converted to a superposition of $\mathrm{LG}_{0,1}$ and $\mathrm{LG}_{0,-1}$ modes [28] as illustrated in Fig. 1(b), where the first index is the radial integer and the second index is the azimuthal index. These two modes have opposite circular polarization, corresponding to two eigenstates of SAM, and their superposition results in a radially polarized beam with zero intensity at its center. The fundamental beam with $\sim 20 \mu \mathrm{J}$ pulse energy is then focused by a $30-\mathrm{cm}$ lens to reach $\sim 1 \mathrm{TW} / \mathrm{cm}^{2}$ on the crystal surface.

Harmonics can be generated within either the perturbative or nonperturbative regimes before irreversible damage. The two limits are distinguished by intensity scaling [Fig. 1(c)] where both the third and fifth harmonics saturate and no longer retain their third and fifth power-law behavior [shown as the black dashed lines in Fig. 1(c)] [29]. The results presented below are obtained in the nonperturbative regime.

During harmonic generation, energy conservation requires that the number of fundamental photons involved in producing harmonics must equal the harmonic order. For instance, in fifth-order harmonic generation shown in Fig. 2(a), five fundamental photons are absorbed and produce one ultraviolet photon emitted at $360 \mathrm{~nm}$. This emitted ultraviolet (UV) photon can only have two possible spin eigenstates, $s=+1$ and $s=-1$, where $s$ is the quantum number of SAM. Therefore, only two possible channels are allowed: either absorbing two left $(s=+1)$ and three right circularly polarized photons $(s=-1)$, or three left $(s=+1)$ and two right $(s=-1)$, as shown in Fig. 2(a) on the right. Any other combinations, for example, those shown on the left, are forbidden, since the final SAM of the UV photon cannot satisfy the condition $\langle s\rangle \leqslant 1$ when spin is conserved.

The total OAM of the emitted harmonic photons for the two allowed processes is equal to the sum of OAM from all involved fundamental photons. In our case, the OAM value for the two processes can only be $l=+1$ and $l=-1$, respectively, where $l$ is the quantum number of the OAM (a)

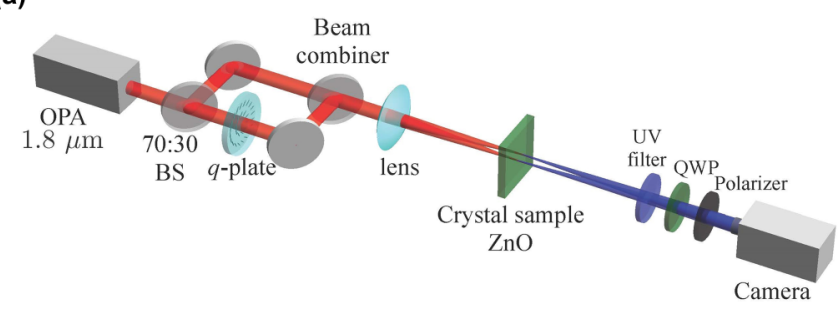

(b)

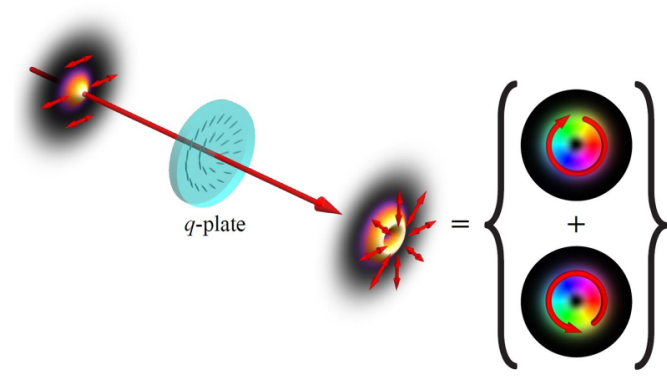

(c)

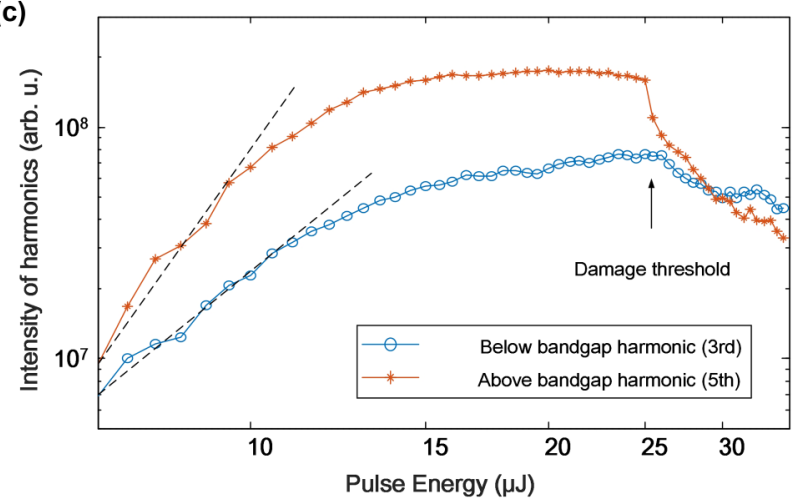

FIG. 1. Generating high-order harmonics under nonperturbative conditions. (a) The experimental setup of generating structured high-harmonic beams from a solid crystal target and interferometric characterization of the OAM on the harmonic beams. A spatially structured beam and a reference beam are both focused on the $\mathrm{ZnO}$ sample with a separation of $200 \mu \mathrm{m}$. (b) A Gaussian beam is converted by a $q$-plate $(q=1 / 2)$ into a superposition of LaguerreGaussian modes with opposite circular polarization states. (c) Intensity of scaling of harmonics yields with respect to different pulse energies of the driving laser pulse.

states. In other words, the OAM of the harmonics equals the OAM of the fundamental. It is the bounded spin states and conservation of angular momentum which selects the OAM value of harmonics. Besides high-order multiphoton process, this selection rule is also held for cascaded low-order nonlinear processes, which may occur in producing the same order harmonics. For example, two fundamental photons mix with a third harmonic photon to generate the fifth harmonic. The emitted photon from the whole cascaded process has $l=s= \pm 1$, since each individual wave mixing conserves spin-orbital angular momentum.

To show that the OAM value of each circularly polarized state is constrained by the spin selection rule, we use a combination of a quarter-wave plate and a linear polarizer to serve as an analyzing element for circular polarization 
(a)

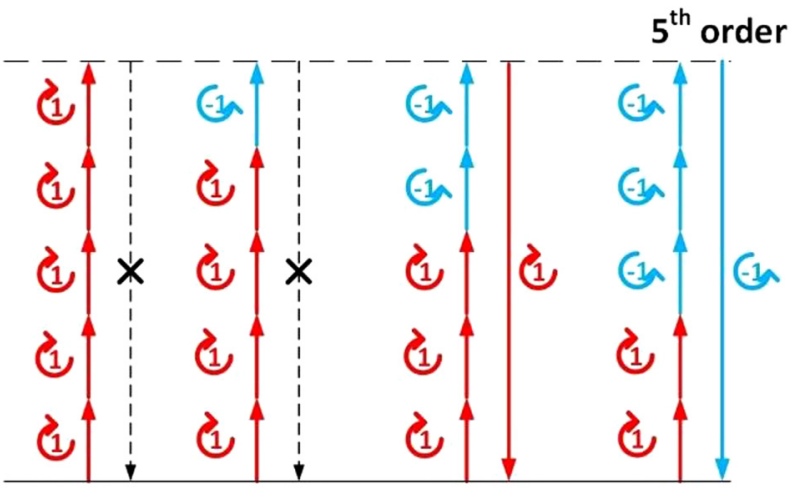

(b)

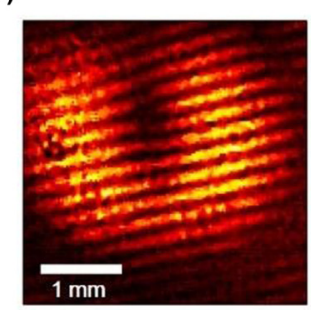

(c)

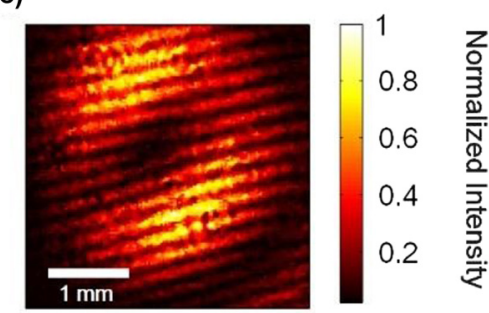

FIG. 2. Control of OAM on fifth-order harmonics by selection rules of SAM. (a) The allowed and forbidden transitions in multiphoton process plotted in the energy diagram. (b) The experimentally observed interference pattern between a reference beam and a lefthanded circularly polarized OAM carrying beam with $l=+1$ in the fifth-order harmonics. (c) The experimentally observed interference pattern between a reference beam and a right-handed circularly polarized OAM carrying beam with $l=-1$ in the fifth-order harmonics. The number of tins on the fork-shaped pattern reveals the OAM value and its orientation indicates the sign of the OAM carried by the left- and right-handed circularly polarized beams.

[30]. Since these two optical components do not change the topology of the wave front, the OAM quantum number can be measured by interference with another plane wave sharing the same polarization state [31]. The beam splitter in Fig. 1(a) reflects a reference fundamental beam which has a Gaussian beam profile without passing through the $q$-plate. This reference beam is also focused on the $\mathrm{ZnO}$ sample and coherently creates a separate independent harmonic source. This reference harmonic beam interferes with the spatially structured harmonic beam on the camera in the far field.

Figures 2(b) and 2(c) show the interferometric results of the $s=+1$ and $s=-1$ state fifth harmonics-above-band-gap harmonics for $\mathrm{ZnO}$. Both results show a fork-shaped pattern and opposite orientations between two circular polarized states. The beam's OAM value can be read from different numbers of fringes from the left side of the singularity point to its right side. The OAM values on the $s= \pm 1$ component are $l= \pm 1$, respectively, which correspond to the OAM of the fundamental beam.

It is possible for the SAM of the optical beam to be transferred to the crystal lattice $[32,33]$ depending on the rotational symmetry of the crystal sample. In our experiment with (0001)-cut free-standing $\mathrm{ZnO}$, such an effect is not excluded. The contribution of these channels can be estimated by the harmonic yield using a circularly driving field. With the $\mathrm{ZnO}$ system, the measured harmonic yields using a circular driving field are below the noise level, which are at least two orders of magnitude weaker than the channels shown in Fig. 2(a) that do not exchange SAM with the crystal lattice. Therefore, the higher-order OAM states are negligible, and the $l= \pm 1$ state dominates the harmonic output.

Symmetry-enabled channels that lead to higher-order OAM modes may be revealed in other strongly coupled systems. For such processes to be important, the material should exhibit an anisotropic harmonic yield with varying orientation of a linearly polarized driving field [15,34].

As a superposition of two equally intense circular states, the driving field is locally linear everywhere at its focus. Thus, the generated harmonic beams should maintain the radially polarized structure of the driving beam. The polarization singularity forces zero intensity at the beam center as shown in Fig. 3(a). To confirm the polarization of the generated harmonic beam, we measure the polarization of the fifth harmonic beam using a linear polarizer, a UV bandpass filter, and a UV enhanced camera, without interfering with the reference beam shown in Fig. 1(a). The intensity profiles of the fifth harmonic after passing through the linear polarizer are shown in Figs. 3(b)-3(d) where we have oriented the linear polarizer at $0^{\circ},-45^{\circ}$, and $90^{\circ}$, respectively. The bright lobes rotate as we change the angle of the linear polarizer. The horizontal/vertical parts of the beam are observed when the optical axis of the linear polarizer is placed horizontally/vertically. This is consistent with the characteristic of a radially polarized beam. From an interferometric point of view, in circular-state bases, the linear polarizer selects a common linear component from the two circular states and lets them interfere collinearly on the camera. The two bright lobes in their intensity illustrate a $2 \times 2 \pi$ phase shift between left and right circular states in the azimuthal direction. This confirms the analysis that one unit of OAM is imparted to the fifth harmonics with opposite signs between the two spin states.

To reach higher photon energies, we use a gas-phase target and a much higher intensity. With a noble gas, the effect of transferring SAM to the nonlinear medium is also eliminated, due to the rotational symmetry of the gas atoms [33]. We use the optical pulse at $800 \mathrm{~nm}$ with $50 \mathrm{fs}$ duration and $0.8 \mathrm{~mJ}$ energy to produce high harmonics from argon gas. The emission extends to the 29th harmonics of $800 \mathrm{~nm}$ with photon energy of $\sim 45 \mathrm{eV}$. The harmonic emission from the gas reproduces a doughnut-shaped intensity distribution from the driving beam, which is shown in Fig. 3(e). The image is recorded directly by a microchannel plate without passing through a dispersive element, to eliminate the polarization selectivity from the measuring system. Therefore, the intensity distribution is an intensity summation of all high harmonics.

However, it is not trivial to separate the two superimposed circularly polarized beams at XUV wavelength and measure their OAM value separately. To link the result from gas and solid targets, we perform equivalently the measurements of Figs. 3(a)-3(d) with a gas-phase target and replace the linear polarizer used for solids by a pair of silver mirrors [35]. The generated high harmonics are passed through both the mirror pair and the XUV imaging grating before being detected by the microchannel plate. The polarization selectivity of the mirror pair and the grating is $\sim 10: 1$. Instead of rotating 

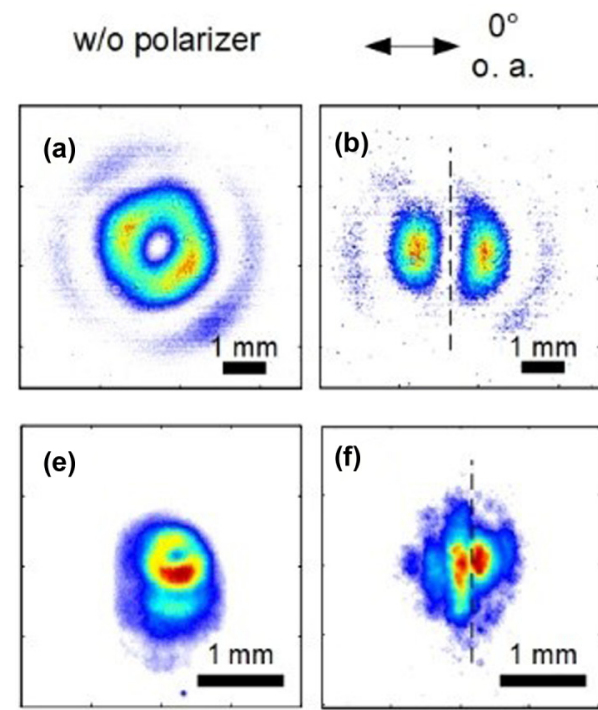
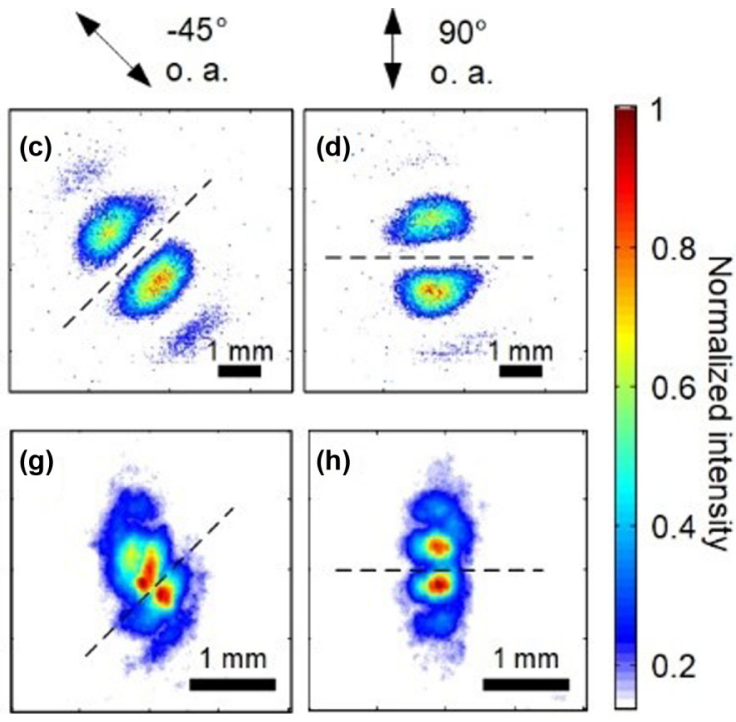

FIG. 3. Characterization of polarization states of high-harmonic beams using a linear polarizer. (a) Intensity profile of generated fifth harmonics from a $\mathrm{ZnO}$ target. (b)-(d) The intensity profiles of fifth harmonic beams from $\mathrm{ZnO}$, with wavelength of $360 \mathrm{~nm}$, after passing through a linear polarizer with its optical axis angle placed at $0^{\circ},-45^{\circ}$ and $90^{\circ}$, respectively. (e) Intensity profile of generated high-order harmonics from an argon gas target. (f)-(h) The intensity profiles of 27 th harmonic beams from argon gas, with a wavelength of $30 \mathrm{~nm}$, after passing through a linear polarizer with its optical axis angle placed at $0^{\circ},-45^{\circ}$, and $90^{\circ}$, respectively. Similar polarization tomographic results indicate that the harmonics generated from the $\mathrm{ZnO}$ crystal and argon gas have similar polarization states.

the mirror pairs, we rotate the incident beam to change the optical axis of the linear polarizer. As shown in Figs. 3(f)3 (h), we see two nodes oriented along different angles as we rotate the driving laser beam. This is the same behavior as we reported in Figs. 3(b)-3(d) for a solid target, and it indicates that controllable OAM is also transferred to a XUV wavelength.

To decouple two superimposed circular states at a XUV wavelength, we propose a bicolor driving approach to isolate a high-harmonic beam with controlled OAM. In that case, the driving field would consist of a fundamental OAM beam with $l=+1, s=+1$ and a second harmonic beam with $l=-1$, $s=-1$. According to the conservation of SAM, there are only two allowed channels for high-harmonic generation, as shown in Fig. 4. Different from the scheme driven by a single-color field, the emissions from these two channels correspond to seventh and eighth harmonic generation. In other words, the two OAM modes are decoupled in energy. Classically, the mixing of the two-color field will result in a threefold symmetric driving field, with a recollision happening every third of a period $[22,36,37]$. Since the two beams carry different phasefront spirals, the threefold trajectories rotate their orientations by $2 \pi$ along the azimuthal direction. This geometric phase gives rise to the phase-front spiral of the generated high harmonics and a recent independent experimental realization of the bicircular scheme for generating vortex beams has been reported [38].

Controllable OAM (or structured polarization states) of short-wavelength radiation will result in tighter optical focusing [39], excite the inner-shell dynamics [40], and probe ultrafast magnetic dynamics [41]. Our study contributes to this growing area of research by exploring the relation between the spin and orbital angular momentum in the up-conversion process under a strong-field condition. Furthermore, we show

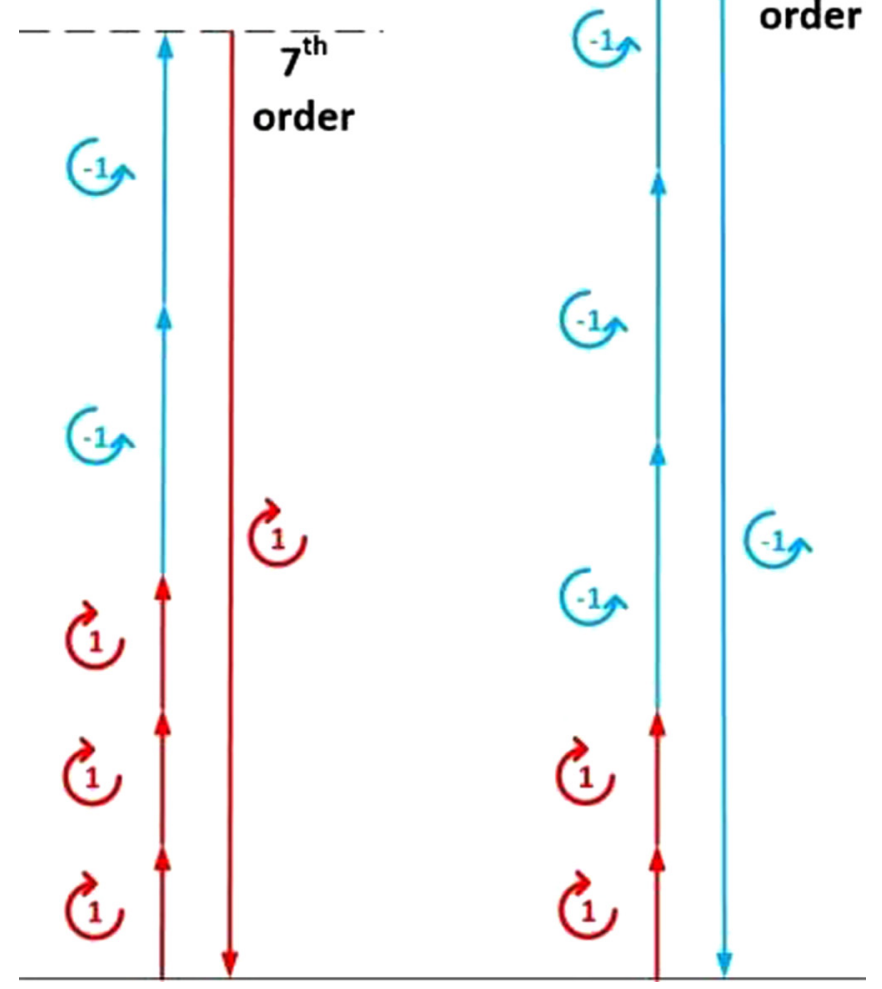

FIG. 4. The energy diagram of the spectrally decoupled OAM mode in high-order harmonics. Two allowed five-photon processes in the two-color scheme are split into seventh and eighth harmonics in the spectrum, whereas the emitted photons are energy degenerate using the same color driving field. 
the potential of solving control problems by linking the two components of the angular momentum. The topological charge is constrained to be equal to that of the fundamental beam. It can therefore be easily manipulated by the conventional optical elements. In addition, the collinear geometry and locally linearly polarized driving field ensure efficient conversion to the harmonic emission, which is crucial for the development of a light source.

In our experiment, both spin and orbital angular momentum are conserved on their own, since the medium that we are interacting with is nearly isotropic and the focusing geometry of the driving laser beam is paraxial. The parametric and coherent nature makes the selection rules robust in both perturbative and nonperturbative conditions. However, exploring the interaction, for example, in waveguide or tightly focused geometry $[42,43]$ where spin and orbital angular momentum can be coupled, may lead to even more complex behavior. Just as other fundamental particles, photons exhibit spinorbit coupling when interacting with matter. The coupling not only exists between spin and orbital angular momentum, it can also happen between the photons and crystal/electronic structures. A strongly coupled solid-phase system may help create electro-optic devices that respond to the polarization and wave front of incident light, or other optical sources, such as polarization/spin controlled soft $\mathrm{x}$ rays for magnetically sensitive probing.

The authors are grateful to Prof. Robert W. Boyd and Prof. Jeff Lundeen from University of Ottawa for their expert advice and helpful criticism during the elaboration of this work. We are also pleased to acknowledge the support of Dr. Andrei Naumov and David Crane from National Research Council of Canada and Yu-Hsuan Wang from University of Ottawa throughout this work. Finally, we acknowledge financial support from U.S. DARPA Topological Excitations in Electronics (TEE) program, Grant No. D18AC00011 and Canada Research Chair (CRC) and Canada Foundation for Innovation (CFI).
[1] Q. Zhan, Adv. Opt. Photonics 1, 1 (2009).

[2] G. Molina-Terriza, J. P. Torres, and L. Torner, Nat. Phys. 3, 305 (2007).

[3] L. Allen, M. W. Beijersbergen, R. J. C. Spreeuw, and J. P. Woerdman, Phys. Rev. A 45, 8185 (1992).

[4] W. Harm, S. Bernet, M. Ritsch-Marte, I. Harder, and N. Lindlein, Opt. Express 23, 413 (2015).

[5] L. Shi, Z. Zhang, A. Cao, X. Luo, and Q. Deng, Opt. Express 23, 8620 (2015).

[6] F. Kong, C. Zhang, F. Bouchard, Z. Li, G. G. Brown, D. H. Ko, T. J. Hammond, L. Arissian, R. W. Boyd, E. Karimi, and P. B. Corkum, Nat. Commun. 8, 14970 (2017).

[7] R. Géneaux, A. Camper, T. Auguste, O. Gobert, J. Caillat, R. Taïeb, and T. Ruchon, Nat. Commun. 7, 12583 (2016).

[8] D. Gauthier, P. R. Ribič, G. Adhikary, A. Camper, C. Chappuis, R. Cucini, L. F. DiMauro, G. Dovillaire, F. Frassetto, R. Géneaux, P. Miotti, L. Poletto, B. Ressel, C. Spezzani, M. Stupar, T. Ruchon, and G. De Ninno, Nat. Commun. 8, 14971 (2017).

[9] E. Hemsing, A. Knyazik, M. Dunning, D. Xiang, A. Marinelli, C. Hast, and J. B. Rosenzweig, Nat. Phys. 9, 549 (2013).

[10] J. Bahrdt, K. Holldack, P. Kuske, R. Müller, M. Scheer, and P. Schmid, Phys. Rev. Lett. 111, 034801 (2013).

[11] G. Gariepy, J. Leach, K. T. Kim, T. J. Hammond, E. Frumker, R. W. Boyd, and P. B. Corkum, Phys. Rev. Lett. 113, 153901 (2014).

[12] L. Rego, J. S. Román, A. Picón, L. Plaja, and C. HernándezGarcía, Phys. Rev. Lett. 117, 163202 (2016).

[13] O. Kfir, P. Grychtol, E. Turgut, R. Knut, D. Zusin, D. Popmintchev, T. Popmintchev, H. Nembach, J. M. Shaw, A. Fleischer, H. Kapteyn, M. Murnane, and O. Cohen, Nat. Photonics 9, 99 (2015).

[14] P. Antoine, A. L'Huillier, M. Lewenstein, P. Salières, and B. Carré, Phys. Rev. A 53, 1725 (1996).

[15] N. Saito, P. Xia, F. Lu, T. Kanai, J. Itatani, and N. Ishii, Optica 4, 1333 (2017).
[16] A. Ferré, C. Handschin, M. Dumergue, F. Burgy, A. Comby, D. Descamps, B. Fabre, G. A. Garcia, R. Géneaux, L. Merceron, E. Mével, L. Nahon, S. Petit, B. Pons, D. Staedter, S. Weber, T. Ruchon, V. Blanchet, and Y. Mairesse, Nat. Photonics 9, 93 (2015).

[17] T. Fan, P. Grychtol, R. Knut, C. Hernández-García, D. D. Hickstein, D. Zusin, C. Gentry, F. J. Dollar, C. A. Mancuso, C. W. Hogle, O. Kfir, D. Legut, K. Carva, J. L. Ellis, K. M. Dorney, C. Chen, O. G. Shpyrko, E. E. Fullerton, O. Cohen, P. M. Oppeneer et al., Proc. Natl. Acad. Sci. USA 112, 14206 (2015).

[18] G. Lambert, B. Vodungbo, J. Gautier, B. Mahieu, V. Malka, S. Sebban, P. Zeitoun, J. Luning, J. Perron, A. Andreev, S. Stremoukhov, F. Ardana-Lamas, A. Dax, C. P. Hauri, A. Sardinha, and M. Fajardo, Nat. Commun. 6, 6167 (2015).

[19] G. Walker, A. S. Arnold, and S. Franke-Arnold, Phys. Rev. Lett. 108, 243601 (2012).

[20] N. V. Bloch, K. Shemer, A. Shapira, R. Shiloh, I. Juwiler, and A. Arie, Phys. Rev. Lett. 108, 233902 (2012).

[21] K. Dholakia, N. B. Simpson, M. J. Padgett, and L. Allen, Phys. Rev. A 54, R3742 (1996).

[22] A. Fleischer, O. Kfir, T. Diskin, P. Sidorenko, and O. Cohen, Nat. Photonics 8, 543 (2014).

[23] W. T. Buono, L. F. C. Moraes, J. A. O. Huguenin, C. E. R. Souza, and A. Z. Khoury, New J. Phys. 16, 093041 (2014).

[24] G. Li, L. Wu, K. F. Li, S. Chen, C. Schlickriede, Z. Xu, S. Huang, W. Li, Y. Liu, E. Y. B. Pun, T. Zentgraf, K. W. Cheah, Y. Luo, and S. Zhang, Nano Lett. 17, 7974 (2017).

[25] H. Larocque, J. Gagnon-Bischoff, F. Bouchard, R. Fickler, J. Upham, R. W. Boyd, and E. Karimi, J. Opt. 18, 124002 (2016).

[26] F. Cardano, E. Karimi, S. Slussarenko, L. Marrucci, C. de Lisio, and E. Santamato, Appl. Opt. 51, C1 (2012).

[27] S. Slussarenko, A. Murauski, T. Du, V. Chigrinov, L. Marrucci, and E. Santamato, Opt. Express 19, 4085 (2011).

[28] E. Karimi, B. Piccirillo, L. Marrucci, and E. Santamato, Opt. Lett. 34, 1225 (2009). 
[29] M. Sivis, M. Taucer, G. Vampa, K. Johnston, A. Staudte, A. Y. Naumov, D. M. Villeneuve, C. Ropers, and P. B. Corkum, Science 357, 303 (2017).

[30] J. Leach, J. Courtial, K. Skeldon, S. M. Barnett, S. FrankeArnold, and M. J. Padgett, Phys. Rev. Lett. 92, 013601 (2004).

[31] M. Harris, C. A. Hill, P. R. Tapster, and J. M. Vaughan, Phys. Rev. A 49, 3119 (1994).

[32] H. J. Simon and N. Bloembergen, Phys. Rev. 171, 1104 (1968).

[33] C. L. Tang and H. Rabin, Phys. Rev. B 3, 4025 (1971).

[34] Y. S. You, D. A. Reis, and S. Ghimire, Nat. Phys. 13, 345 (2017).

[35] J. Levesque, Y. Mairesse, N. Dudovich, H. Pépin, J.-C. Kieffer, P. B. Corkum, and D. M. Villeneuve, Phys. Rev. Lett. 99, 243001 (2007).

[36] H. Eichmann, A. Egbert, S. Nolte, C. Momma, B. Wellegehausen, W. Becker, S. Long, and J. K. McIver, Phys. Rev. A 51, R3414 (1995).
[37] D. B. Milošević and W. Becker, Phys. Rev. A 62, 011403(R) (2000).

[38] K. M. Dorney, L. Rego, N. J. Brooks, J. San Román, C.-T. Liao, J. L. Ellis, D. Zusin, C. Gentry, Q. L. Nguyen, J. M. Shaw, A. Picón, L. Plaja, H. C. Kapteyn, M. M. Murnane, and C. Hernández-García, Nat. Photonics 13, 123 (2019).

[39] R. Dorn, S. Quabis, and G. Leuchs, Phys. Rev. Lett. 91, 233901 (2003).

[40] A. D. Shiner, B. E. Schmidt, C. Trallero-Herrero, H. J. Wörner, S. Patchkovskii, P. B. Corkum, J.-C. Kieffer, F. Légaré, and D. M. Villeneuve, Nat. Phys. 7, 464 (2011).

[41] A. V. Kimel, A. Kirilyuk, P. A. Usachev, R. V. Pisarev, A. M. Balbashov, and T. Rasing, Nature (London) 435, 655 (2005).

[42] K. Y. Bliokh, E. A. Ostrovskaya, M. A. Alonso, O. G. Rodríguez-Herrera, D. Lara, and C. Dainty, Opt. Express 19, 26132 (2011).

[43] K. Y. Bliokh, F. J. Rodríguez-Fortuño, F. Nori, and A. V. Zayats, Nat. Photonics 9, 796 (2015). 